\title{
Black holes as fundamental strings: Comparing the absorption of scalars
}

\author{
Roberto Emparan* \\ Department of Physics, University of California, Santa Barbara, California 93106
}

(Received 5 May 1997)

\begin{abstract}
The recently proposed "correspondence principle" of Horowitz and Polchinski provides a concrete means to relate (among others) black holes with electric Neveu-Schwarz-Neveu-Schwarz charges to fundamental strings and correctly match their entropies. We further test this correspondence by examining the greybody factors in the absorption rates of neutral, minimally coupled scalars by a near extremal black hole. Perhaps surprisingly, the results disagree in general with the absorption by weakly coupled strings. Though this does not disprove the correspondence, it indicates that it might not be simple in this region of the black hole parameter space. [S0556-2821(97)00618-8]

PACS number(s): 04.70.Dy
\end{abstract}

\section{INTRODUCTION}

During the past year there has been impressive progress in our understanding of the microscopic description of black holes [1] (see [2,3] for reviews and further references). For the case that is best understood, a description has emerged for the weak coupling dynamics of a five-dimensional black hole near extremality in terms of an "effective string." The latter is in fact a bound state of $D$-strings restricted to move inside a $D$-fivebrane, and which is excited above the Bogomol'nyi-Prasad-Sommerfield (BPS) state by having both left- and right-moving momenta running along the string (in a dilute gas regime) [4,5]. This model precisely reproduces the Bekenstein-Hawking entropy of the black hole in terms of the degeneracy of states of the effective string, and Hawking radiation comes about as the emission of closed string states resulting from annihilation of pairs of left and right moving quanta. This effective description has turned out to be surprisingly successful. Not only the entropy can be correctly reproduced, but also details of the scalar emission rates computed from the effective string show precise agreement with the spectrum of Hawking radiation. This agreement is correct including normalization factors [6] and extends to the level of black hole greybody profiles [7]. ${ }^{1} \mathrm{~A}$ possible explanation for this success has been proposed in [12].

Still within the near-extremal realm, it has been shown in [13] that when one adds $D$-string-antistring pairs to the bound state described above, the black hole entropy can be correctly accounted for by the states of a noncritical Polyakov string with its effective tension and central charge constrained by the $D$-fivebrane background. In this regime, however, it is unclear how strong coupling effects can be avoided. Moreover, the details of spectroscopy show only partial agreement, and deviations occur at the level of leading order corrections to the absorption cross section at low energies [14].

\footnotetext{
*Electronic address: emparan@ cosmic1.physics.ucsb.edu

${ }^{1}$ The agreement has been further reinforced in [8]. However, it should be noted that disagreement has also been found in certain specific regimes [9-11].
}

Pushing the picture further along these lines, one could expect to find a description of the (five-dimensional) neutral Schwarzschild black hole as a bound state of a number of $D$-fivebranes, $D$-strings, and momenta together with an equal number of their antiexcitations [15]. However, it is not clear at all how to compute reliably the degeneracy of states for such a system, or even to justify or understand, in general, why the brane-antibrane pairs should not annihilate.

A rather different approach for understanding the entropy of the Schwarzschild black hole was initiated by Susskind a few years back [16]. Here, the working hypothesis is that the only objects needed to account for the degrees of freedom of a neutral black hole are fundamental strings - and not, in particular, $D$-branes. In this picture, if we start at strong coupling with a state that looks like a black hole, then as we decrease the coupling the horizon shrinks. Eventually, the state is better described as a long, highly excited string. This conjecture has been carried further in $[17-19] .^{2}$ The obvious difficulty with this approach, namely, the different growth of the number of states with a given energy for black holes and strings, has been recently solved in a convincing manner by Horowitz and Polchinski in [19]. The key observation is that we should not expect the mass of a certain black hole statewhich is fixed in Planck units-to be equal to that of the corresponding string state-constant in string units-for arbitrary values of the string coupling constant $g$. Rather, the correspondence between black hole and string parameters should be naturally made at the value of $g$ for which the transition between both descriptions takes place. There is a shortcoming here in that we do not know how to determine precisely at which value of the coupling this transition happens. Hence this principle only allows one to relate quantities up to factors of order 1 . Nevertheless, its range of applicability is wider than for other approaches - most of which it subsumes - and it has been shown in [19] to yield the correct dependence of the entropy on black hole parameters for a large number of nontrivial cases, such as, in particular, black holes with charges corresponding to the winding and mo-

\footnotetext{
${ }^{2}$ See [20] for another, black-hole-guided, approach to a string model for black holes arbitrarily far from extremality.
} 
mentum numbers of a fundamental string.

A key premise of the Horowitz-Polchinski correspondence principle is that, when the strongly coupled black hole and the weakly coupled string are taken to the transition point, the mass of neither of them changes by a large factor. In practice, this means that, at the matching point, the classical black hole mass can be taken to be approximately equal to the energy of a string whose levels are determined according to the spectrum of a free (or very weakly coupled) string. Neither the finite coupling corrections to the string mass spectrum, nor the string-size modifications to the black hole geometry introduce large factors that could alter the matching of masses and entropies at the transition point. Our aim in this paper is to test if this simplest classical-black-holefree-string correspondence is enough to account for details of the radiation such as greybody filtering. This is particularly interesting because, as was revealed in [7], greybody factors encode information on the excitation spectrum of the string. For technical reasons, the computations in the black hole side will have to be restricted to near extremal configurations in five and four dimensions. The results we find indicate that, at least in the region of the black hole parameter space that we are able to probe, the simplest model for the correspondence does not seem to account correctly for the details of the spectrum. There is some evidence that the discrepancy could be traced to the fact that, for the class of black holes under consideration, the near extremal state at the matching point actually is not infinitesimally close to the BPS limit.

The paper is organized as follows. In Sec. II we review the calculation of scalar absorption by five-dimensional (5D) and 4D near extremal black holes which will be of use later on. Then, in Sec. III we make use of the correspondence principle to compare these results with the absorption rates of neutral scalars by fundamental strings previously computed in [18]. We give a simple argument which shows that, quite generally, disagreement is found. We conclude in Sec. IV with a discussion of the results and their implications.

\section{ABSORPTION RATES FOR NEAR EXTREMAL FIVE- AND FOUR-DIMENSIONAL BLACK HOLES}

The absorption cross sections, including greybody factors, of minimally coupled scalars by a near extremal black hole in five and four dimensions have been computed in great generality in [14]. These results will be needed later, so we find it useful to describe how they are obtained.

We start with the nonrotating nonextremal fivedimensional black hole solution of string theory $[21,15]$ which takes the form, in the Einstein conformal gauge,

$$
d s^{2}=-\frac{h}{f^{2 / 3}} d t^{2}+f^{1 / 3}\left(\frac{d r^{2}}{h}+r^{2} d \Omega_{3}^{2}\right)
$$

with

$$
\begin{gathered}
h=1-\frac{r_{0}^{2}}{r^{2}}, \quad f=f_{1} f_{2} f_{3}, \\
f_{i}=1+\frac{r_{i}^{2}}{r^{2}}, \quad r_{i}^{2}=r_{0}^{2} \sinh ^{2} \sigma_{i}, \quad i=1,2,3 .
\end{gathered}
$$

This solution admits a variety of embeddings in several different compactifications of any of the superstring (and $M$ ) theories. The characteristic radii $r_{i}$ are associated with three different types of charges, and their interpretation [as, e.g., Kaluza-Klein (KK) momentum, winding, Ramond-Ramond (RR) electric or magnetic charge, etc.], depends on the embedding. In particular, for the main purposes of this paper (Sec. III) we shall only need to consider two nonzero charges. Nonetheless, for the moment we leave the number of charges and their interpretation otherwise unspecified, but take the radii to be ordered as

$$
r_{1} \geqslant r_{2} \geqslant r_{3} \text {. }
$$

Besides the "nonextremality parameter' $r_{0}$, one works with the three radii, $r_{i}$, or alternatively, the associated hyperbolic angles, $\sigma_{i}$, depending on convenience.

The mass, entropy, and Hawking temperature of the black hole are

$$
\begin{gathered}
M=\frac{\pi r_{0}^{2}}{8 G_{5}}\left(\cosh 2 \sigma_{1}+\cosh 2 \sigma_{2}+\cosh 2 \sigma_{3}\right), \\
S=\frac{\pi^{2} r_{0}^{3}}{2 G_{5}} \cosh \sigma_{1} \cosh \sigma_{2} \cosh \sigma_{3}, \\
T_{H}^{-1}=2 \pi r_{0} \cosh \sigma_{1} \cosh \sigma_{2} \cosh \sigma_{3} .
\end{gathered}
$$

The black hole is taken to near extremality by having at least one large charge, say $r_{0} \ll r_{1}$, so that $r_{1}=r_{0} \sinh \sigma_{1} \approx r_{0} \cosh \sigma_{1}$. The other two radii (charges) can be as well large or small. The scattering will be restricted to $s$ waves, higher partial waves being suppressed by centrifugal potential barriers at frequencies such that $\omega r_{0} \ll 1$ and $\omega r_{1}<2$ [14]. Although it would not be difficult to include higher angular momenta, our primary interest will be on a range of low enough frequencies specified by $\omega r_{1} \ll 1$.

The wave equation for a minimally coupled, spherically symmetric scalar field $\Phi(r, t)=e^{-i \omega t} R(r)$ in this black hole background takes the form

$$
\left(\frac{h}{r^{3}} \frac{d}{d r} h r^{3} \frac{d}{d r}+\omega^{2} f\right) R=0 .
$$

An alternative set of four parameters characterizing the black hole is useful when dealing with the wave equation. These are

$$
\begin{gathered}
D=\frac{\omega^{2}}{4 r_{0}^{4}} r_{1}^{2} r_{2}^{2} r_{3}^{2}, \\
C=\frac{\omega^{2}}{4 r_{0}^{2}}\left(r_{1}^{2} r_{2}^{2}+r_{1}^{2} r_{3}^{2}+r_{2}^{2} r_{3}^{2}\right), \\
E=\frac{\omega^{2}}{4}\left(r_{1}^{2}+r_{2}^{2}+r_{3}^{2}\right), \\
E_{2}=\frac{\omega^{2}}{4} r_{0}^{2} .
\end{gathered}
$$


It is also convenient to define the variable $z=h(r)$ in terms of which the wave equation takes the form

$$
\left(z \frac{d}{d z}\right)^{2} R+\left(D+\frac{C}{(1-z)}+\frac{E}{(1-z)^{2}}+\frac{E_{2}}{(1-z)^{3}}\right) R=0 .
$$

The latter is still the exact wave equation for the s-wave scalar. Since, in general, it is not possible to solve it analytically for arbitrary values of $r$, one needs to resort to approximate methods. ${ }^{3}$ The traditional way to deal with the problem [22-25,27,6,7] has been to, first, solve the equations in two different regions: (I) near the horizon; (II) far from the black hole, and then match the solutions at some point in between, or by means of an intermediate region. For near-extremal black holes of the sort under consideration, Rajaraman has studied in detail the definition of regions (I) and (II), and has settled the question of the existence of an overlap region and the matching of solutions [26].

Boundary conditions are imposed at the black hole horizon, by requiring the wave to be purely ingoing into the black hole. The matching of solutions in (I) and (II) then allows one to determine the ratio at asymptotic infinity of the amplitudes of radially ingoing waves to those outcoming, and hence the luminosity of the black hole.

Very close to the horizon $(z \rightarrow 0)$ an approximate solution can be found if we neglect the $z$ dependence in the nonderivative term in Eq. (2.7). In this way one finds

$$
R_{\mathrm{I}} \simeq e^{ \pm i \sqrt{D+C+E+E_{2}} \ln z}=\exp \left( \pm \frac{i \omega A_{b h}}{4 \pi^{2} r_{0}^{2}} \ln z\right),
$$

where the $+(-)$ solution is outcoming (ingoing) at the horizon. This is essentially the form of the solution used in $[25,24]$, and can be seen to yield correctly the leading term, at low frequencies, of the absorption cross section as equal to the horizon area of the black hole. However, in order to keep further dependence on $z$ and be able to enlarge the distance within which the solution is valid, it is found adequate $[7,14]$ to try the ansatz $R_{\mathrm{I}}=z^{\alpha}(1-z)^{\beta} F(z)$. In the region where $E_{2}$ can be dropped a solution can be found with $F$ a hypergeometric function. Explicitly,

$$
R_{\mathrm{I}}=z^{\alpha}(1-z)^{\beta} F(\alpha+\beta+i \sqrt{D}, \alpha+\beta-i \sqrt{D} ; 1+2 \alpha ; z)
$$

with

$$
\alpha=-i \sqrt{D+C+E}, \quad \beta=\frac{1}{2}(1-\sqrt{1-4 E}) .
$$

\footnotetext{
${ }^{3}$ However, it must be noted that for the extremal black hole with only one single type of charge it is indeed possible to solve analytically the wave equation exactly, everywhere and for arbitrary frequencies (and also include higher partial waves), in terms of Bessel functions. For this black hole, though, the horizon is a singularity of zero area. In a similar way, for the extreme four-dimensional black hole with two charges the exact solution can be found in terms of Coulomb wave functions.
}

Neglection of the $E_{2}$ term is certainly justified provided $r \ll r_{1}$.

The solution has been completely fixed, up to arbitrary global wave function normalization, by demanding that, very close to the horizon, the solution behaves as the ingoing wave (2.8). We also need the limiting form of $R_{\mathrm{I}}$ for large $r$, i.e., $z \rightarrow 1$. This is

$$
R_{\mathrm{I}} \sim(1-z)^{\beta} \frac{\Gamma(1+2 \alpha) \Gamma(1-2 \beta)}{\Gamma(1+\alpha-i \sqrt{D}-\beta) \Gamma(1+\alpha+i \sqrt{D}-\beta)} .
$$

To analyze the equation far from the horizon it is convenient to define $\rho=\omega r$ and $R=\psi(\rho) / \rho$. If $r \gg r_{0}$ we can approximate $h \simeq 1$. The wave equation becomes

$$
\rho^{2} \psi^{\prime \prime}+\rho \psi^{\prime}-\left(1-\rho^{2} f\right) \psi=0 .
$$

Approximate now $1-\rho^{2} f \approx 1-4 E-\rho^{2}$, which requires $1 \gg \omega^{4} r_{1}^{2} r_{2}^{2} / \rho^{2}$, i.e., $r \gg \omega r_{1} r_{2}$. Upon doing so we find a Bessel equation for $\psi$, and the general solution can be expressed in terms of Bessel functions $J_{\nu}(\omega r)$ as

$$
R_{\mathrm{II}}=\frac{A}{\omega r} J_{1-2 \beta}(\omega r)+\frac{B}{\omega r} J_{2 \beta-1}(\omega r) .
$$

For small $\rho$ the behavior of $R_{\mathrm{II}}$ is found from

$$
\begin{gathered}
\frac{1}{\rho} J_{1-2 \beta}(\rho) \sim \frac{1}{2}\left(\frac{2}{\rho}\right)^{2 \beta} \frac{1}{\Gamma(2-2 \beta)}, \\
\frac{1}{\rho} J_{2 \beta-1}(\rho) \sim \frac{2}{\rho^{2}}\left(\frac{\rho}{2}\right)^{2 \beta} \frac{1}{\Gamma(2 \beta)} .
\end{gathered}
$$

From here we see that the wave $J_{1-2 \beta}(\omega r) /(\omega r)$ behaves in this region in exactly the same way as the wave $R_{\mathrm{I}}$ for $r \gg r_{0}$, Eq. (2.11). Moreover, $R_{\mathrm{I}}$ is definitely valid up to $r \ll r_{1}$ (in fact, up to $r \ll 1 / \omega$ [26]), and $R_{\mathrm{II}}$ down to $r \gg \omega r_{1} r_{2}$. Hence, there exists an overlap region where the matching can be done. The coefficients in Eq. (2.13) are determined as $B=0^{4}$ and

$$
A=2\left(\frac{\omega r_{0}}{2}\right)^{2 \beta} \frac{\Gamma(1+2 \alpha) \Gamma(1-2 \beta) \Gamma(2-2 \beta)}{\Gamma(1+\alpha-i \sqrt{D}-\beta) \Gamma(1+\alpha+i \sqrt{D}-\beta)} .
$$

This fixes, up to global wave function normalization, the solution to the wave equation with the required boundary conditions at the horizon.

Having constructed this solution for $s$-wave scattering, and in the approximation where we neglect higher partial waves, the plane wave absorption cross section can be finally found to be

$$
\sigma_{\mathrm{abs}}=\frac{16 \pi^{2} i \alpha r_{0}^{2}}{\omega|A|^{2}}
$$

\footnotetext{
${ }^{4}$ More precisely, $B / A$ is small when $\beta$ is small. We thank J. Traschen for discussions on this point.
} 
This form of the solution was first found in [14].

The expression for $\sigma_{\text {abs }}$ is much more amenable to physical interpretation if $|A|^{2}$ is expanded for small $\beta$. Still, since

$$
i \alpha \sim \max \left\{\omega r_{1}, \frac{\omega r_{1} r_{2}}{r_{0}}, \frac{\omega r_{1} r_{2} r_{3}}{r_{0}^{2}}\right\},
$$

it is possible to keep $|\alpha|$ of order one as long as there are, at least, two large charges. ${ }^{5}$ In this regime (which is the one analyzed in [7]) straightforward algebra yields

$$
\begin{aligned}
\sigma_{\mathrm{abs}}= & \frac{4 \pi^{3} r_{0}^{2}\left|\alpha^{2}+D\right|}{\omega} \frac{e^{4 \pi i \alpha}-1}{\left(e^{2 \pi i(\alpha+i \sqrt{D})}-1\right)\left(e^{2 \pi i(\alpha-i \sqrt{D})}-1\right)} \\
& \times\left[1+O\left(\omega^{2} r_{1}^{2}\right)\right] .
\end{aligned}
$$

If there is just one large charge, then not only $\beta$ but also $|\alpha|$ and $\sqrt{D}$ have to be regarded as very small. The absorption cross section should be written now as

$$
\begin{aligned}
\sigma_{\text {abs }}= & \frac{4 \pi^{2} r_{0}^{2} i \alpha}{\omega}\left\{1+\frac{\pi^{2}}{3}\left|\alpha^{2}+D\right|-4 \beta\left[\ln \left(\frac{\omega r_{0}}{2}\right)-1+\gamma\right]\right. \\
& \left.+O\left(\omega^{4} r_{1}^{4}\right)\right\},
\end{aligned}
$$

where $\gamma$ is the Euler-Mascheroni constant. The logarithmic term comes from expanding the term $\left(\omega r_{0} / 2\right)^{4 \beta}$ in $|A|^{2}$.

Let us rewrite these expressions for $\sigma_{\text {abs }}$ in terms of physical quantities. Given that we always assume that there is at least one large charge, one has

$$
\begin{aligned}
i \alpha & =\frac{\omega A_{\mathrm{BH}}}{4 \pi^{2} r_{0}^{2}}\left[1+O\left(r_{0}^{2} / r_{1}^{2}\right)\right] \\
& =\frac{\omega}{4 \pi T_{H}}\left[1+O\left(r_{0}^{2} / r_{1}^{2}\right)\right] .
\end{aligned}
$$

From here and Eq. (2.20) one finds that, for $\omega \rightarrow 0$, the absorption cross section is strictly equal to the black hole area, $\sigma_{\mathrm{abs}}=A_{\mathrm{BH}}+O(\omega)[25,28]$.

Consider now small but nonvanishing frequencies. From the second expression for $i \alpha$ in Eq. (2.21) we see that the factor $\left(e^{4 \pi i \alpha}-1\right)$ in Eq. (2.19) is precisely the Planckian factor for the Hawking radiation. This suggests to define, in a similar way, two other "left" and "right" temperatures as

$$
\begin{aligned}
T_{R, L}^{-1} & =\frac{4 \pi}{\omega}(i \alpha \pm \sqrt{D}) \\
& \simeq 2 \pi r_{1} \cosh \left(\sigma_{2} \pm \sigma_{3}\right) .
\end{aligned}
$$

The last expression is found bearing in mind that $r_{1}$ is a large radius. The absorption cross section (2.19) presents now the suggestive form

\footnotetext{
${ }^{5}$ But keep in mind that they need not be of the same order; i.e., we
} can have $r_{1} \gg r_{2} \gg r_{0}$.

$$
\begin{aligned}
\sigma_{\mathrm{abs}}= & A_{\mathrm{BH}} \frac{\omega}{2\left(T_{R}+T_{L}\right)} \frac{e^{\omega / T_{H}}-1}{\left(e^{\omega / 2 T_{R}}-1\right)\left(e^{\omega / 2 T_{L}}-1\right)} \\
& \times\left[1+O\left(\omega^{2} r_{1}^{2}\right)\right] .
\end{aligned}
$$

On the other hand, if there is only one large charge, $\left(\omega / T_{L, R, H}\right)^{2} \sim E \ll 1$, the result is

$$
\begin{aligned}
\sigma_{\mathrm{abs}}= & A_{\mathrm{BH}}\left\{1+\frac{\omega^{2}}{48} \frac{1}{T_{R} T_{L}}-\omega^{2} r_{1}^{2}\left[\ln \left(\frac{\omega r_{0}}{2}\right)-1+\gamma\right]\right. \\
& \left.+O\left(\omega^{4} r_{1}^{4}\right)\right\} .
\end{aligned}
$$

Notice that the most relevant correction term is logarithmic, $\sim \beta \ln \left(\omega r_{0}\right)$. Since $\omega r_{0} \ll 1$, this can be quite significant and is larger than the terms coming from the exponential greybody factors.

The greybody factors of thermal form in Eq. (2.23) were found by Maldacena and Strominger [7], who also showed that $T_{R, L}$ correspond exactly to effective temperatures for the left- and right-moving vibrations of the "effective string" model for the five-dimensional black hole with two large $D$-brane charges. The case when just one of the charges is large has been solved more recently in [14], and the logarithmic correction noted. Its absence from the simplest string calculation has been interpreted as suggesting that the effective string model should be modified in the corresponding region of parameter space.

In four dimensions we consider the nonrotating nonextremal black hole with four charges,

$$
d s^{2}=-\frac{h}{f^{1 / 2}} d t^{2}+f^{1 / 2}\left(\frac{d r^{2}}{h}+r^{2} d \Omega_{2}^{2}\right),
$$

with

$$
h=1-\frac{r_{0}}{r}, \quad f=f_{1} f_{2} f_{3} f_{4},
$$

$$
f_{i}=1+\frac{r_{i}}{r}, \quad r_{i}=r_{0} \sinh ^{2} \sigma_{i}, \quad i=1,2,3,4,
$$

and mass, entropy and temperature,

$$
\begin{gathered}
M=\frac{r_{0}}{8 G_{4}} \sum_{i=1}^{4} \cosh 2 \sigma_{i}, \\
S=\frac{\pi r_{0}^{2}}{G_{4}} \prod_{i=1}^{4} \cosh \sigma_{i}, \\
T_{H}^{-1}=4 \pi r_{0} \prod_{i=1}^{4} \cosh \sigma_{i} .
\end{gathered}
$$

As before, we order the radial parameters as

$$
r_{1} \geqslant r_{2} \geqslant r_{3} \geqslant r_{4} \text {. }
$$

The analysis of absorption rates, carried out in [14], is quite similar to that for the 5D black hole. However, the 4D 
case differs from the 5D case in two crucial respects: first, in order to be able to solve the equations one needs to restrict to the case with at least two large charges:

$$
r_{1}, r_{2} \gg r_{0}
$$

Thus, we are not able to deal with the most general near extremal black holes in four dimensions.

Second, the leading correction at low frequencies is found to be linear in $\omega$, in contrast to the 5D result where corrections start to appear at order $\omega^{2}$. As a consequence, at low enough frequencies the linear term completely masks the (quadratic) terms coming from expanding the exponential thermal factors, as well as the logarithmic correction terms found in the previous section. More importantly, such a correction (also found, in a slightly different context, in [29]) does not seem to appear in the results obtained using the effective string model for the 4D black hole [14].

Near the horizon the analysis is very closely similar to that in five dimensions. On the other hand, far from the horizon the wave equation can be solved in terms of Coulomb wave functions, $F_{L}(\eta, \omega r)$, (with noninteger $L$ ) as

$$
R_{\mathrm{II}}=\frac{A}{\omega r} F_{-\beta}(\eta, \omega r)+\frac{B}{\omega r} F_{\beta-1}(\eta, \omega r),
$$

where

$$
\eta \equiv-\frac{\omega}{2}\left(r_{1}+r_{2}+r_{3}+r_{4}\right)
$$

and $\beta \approx \omega^{2} r_{1} r_{2}$ are small quantities.

For small $\omega r$ the functions $F_{L}(\eta, \omega r)$ have again a powerlike dependence that enables $R_{\mathrm{II}}$ in Eq. (2.30) to be easily matched to the near region solution (2.11). Define now

$$
T_{R, L}^{-1}=4 \pi \sqrt{r_{1} r_{2}} \cosh \left(\sigma_{3} \pm \sigma_{4}\right)
$$

When $\omega / T_{L, R, H} \sim 1$ (which now requires at least three large charges), the absorption cross section takes the form

$$
\begin{aligned}
\sigma_{\mathrm{abs}}= & A_{\mathrm{BH}} \frac{\omega}{2\left(T_{R}+T_{L}\right)} \frac{e^{\omega / T_{H}}-1}{\left(e^{\omega / 2 T_{R}}-1\right)\left(e^{\omega / 2 T_{L}}-1\right)} \\
& \times\left[1+O\left(\omega r_{1}\right)\right] .
\end{aligned}
$$

When there are only two large charges $\omega / T_{R, L}$ are small, and the result would be

$$
\sigma_{\mathrm{abs}}=A_{\mathrm{BH}}\left(1-\pi \eta+\frac{\omega^{2}}{48} \frac{1}{T_{R} T_{L}}+\cdots\right) .
$$

Since $\eta$ is already linear in $\omega r_{1}$ we have neglected the terms proportional to $\beta$, similar to those found in five dimensions. Indeed, given that $\omega^{2} / T_{R} T_{L} \sim \omega^{2} r_{1} r_{2}$, in this regime the "temperature dependent" corrections are negligible to the order we are working. Thus, for two large charges the leading corrections in four and five dimensions are very different.

\section{FUNDAMENTAL STRINGS VS BLACK HOLES}

Fundamental strings can carry two kinds of charges, namely electric Neveu-Schwarz-Neveu-Schwarz (NS-NS) charges, associated with the momentum and winding modes of the string. Black holes with these same quantum numbers can be readily constructed [30]. Since, in general, string states with given winding and momentum are highly degenerate, one would expect a relation with the BekensteinHawking entropy of the black hole to be viable. This is not straightforward. For one thing, a secure starting point for the identification would be a supersymmetry-protected BPS state, i.e., the extremal black hole. But for the NS-NS electric black holes the horizon becomes a zero-area singularity in the extremal limit, suggesting that stringy corrections to the geometry should be relevant in its vicinity. Sen has invoked these corrections to argue that a stretched horizon of string size should be present, whose area reproduces the degeneracy of string BPS states [31].

The correspondence principle proposed in [19] provides a concrete way to relate strings and black holes (in any dimension $D \geqslant 4$ ) arbitrarily away from extremality, and correctly obtain, up to factors of order 1, the Bekenstein-Hawking entropy by counting string states. As explained in [19], the comparison between the black hole picture at strong coupling, on the one hand, and the string to which it evolves at weak coupling on the other hand, should be naturally made at the value of the string coupling where the former description yields way to the latter. This should happen when the curvature of the black hole geometry (in the string conformal frame) reaches the string size. At this point, the mass and degeneracy of states of the black hole can be matched, up to factors of order 1, to those of the string.

The string to which the black hole evolves is a highly excited one, in a thermal state. Its decay by annihilation of left- and right-moving oscillations reproduces the thermal character of Hawking radiation. However, the entropy, or equivalently, the Hawking temperature, only conveys information about the total excitation level of the string. In particular, it is not possible to tell from the entropy alone any differences between the excitations of left- and right-moving oscillators. Remarkably, as first discussed in [7], it turns out that the radiation emitted from the black hole actually encodes such information in the greybody filtering of the Planckian spectrum. It is this sort of analysis what we want to perform here.

Let us, first, briefly review how the correspondence between a fundamental string and the black hole goes in the five-dimensional case. Consider a string moving on a circle of radius $R$, carrying $n_{p}$ and $n_{w}$ units of momentum and winding, respectively. If $N_{R, L}$ are the right and left oscillation level numbers, then the mass levels of the free string are given by

$$
\begin{aligned}
M^{2} & =\left(\frac{n_{p}}{R}+\frac{n_{w} R}{\alpha^{\prime}}\right)^{2}+\frac{4}{\alpha^{\prime}} N_{R} \\
& =\left(\frac{n_{p}}{R}-\frac{n_{w} R}{\alpha^{\prime}}\right)^{2}+\frac{4}{\alpha^{\prime}} N_{L} .
\end{aligned}
$$

A six-dimensional black string can be constructed with these quantum numbers [32]. Its metric, in the string frame, is 


$$
\begin{aligned}
d s_{(6)}^{2}= & -\frac{h}{f_{w} f_{p}} d t^{2}+\frac{f_{p}}{f_{w}}\left(d z-\frac{r_{0}^{2} \sinh 2 \sigma_{p}}{2 r^{2} f_{p}} d t\right)^{2}+\frac{d r^{2}}{h} \\
& +r^{2} d \Omega_{3}^{2},
\end{aligned}
$$

where $f_{p}, f_{w}, h$ are functions like those in Eq. (2.2). The momentum and winding NS-NS charges are identified as

$$
n_{p}=\frac{\pi R}{8 G_{5}} r_{0}^{2} \sinh 2 \sigma_{p}, \quad n_{w}=\frac{\pi \alpha^{\prime}}{8 G_{5} R} r_{0}^{2} \sinh 2 \sigma_{w} .
$$

The momentum actually results from boosting along $(t, z)$ a string which initially has $n_{p}=0$.

We have expressed these charges in terms of the fivedimensional Newton constant, which can be obtained as $G_{5}=\pi g^{2}\left(\alpha^{\prime}\right)^{4} /(4 R V)$, with $g$ the 10-dimensional string coupling, and $(2 \pi)^{4} V$ a compactified four-volume. The reason for this choice is that upon reduction along the wrapping direction of the string one obtains the five-dimensional black hole of Sec. II with two nonzero charges. In our identification with black hole parameters, we take $r_{w} \equiv r_{1}$ to be the radius associated with the winding charge, $r_{p} \equiv r_{2}\left(\leqslant r_{1}\right)$ associated with the momentum charge (the case $r_{p} \geqslant r_{w}$ is $T$ dual to this one), and $r_{3}=0$.

If we keep the charges fixed, then as we decrease the coupling $g$ the horizon radius $r_{0}$ becomes smaller. Following [19], the string-frame curvature reaches the string scale when $r_{0} \sim \sqrt{\alpha^{\prime}}$. At this point the mass of the string in Eq. (3.1) can be set equal to that of the black hole (2.4). This allows one to determine the oscillation levels $N_{R}, N_{L}$. These could be either of similar magnitude, or one much larger than the other. But remarkably, as noted in [19], the sum $\sqrt{N_{L}}+\sqrt{N_{R}}$, which corresponds to the string degeneracy of states, is, up to a factor of order 1, independent of the relative size of the summands,

$$
\begin{aligned}
S_{\mathrm{st}} & \sim \sqrt{N_{L}}+\sqrt{N_{R}} \\
& \sim \frac{\left(\alpha^{\prime}\right)^{3 / 2}}{G_{5}} \cosh \sigma_{w} \cosh \sigma_{p} \\
& \sim S_{\mathrm{BH}},
\end{aligned}
$$

and therefore the string entropy correctly reproduces, within the accuracy of the correspondence principle and for arbitrary momentum and winding numbers, the BekensteinHawking entropy of the black hole (2.4) at the matching point.

Our task now is to test the absorption rates of scalar particles. The comparison has to be confined to near extremal situations, where we can use the results of the previous sections for the classical absorption by the black hole. For future reference, the entropy and mass of such black holes at the matching point is

$$
S_{\mathrm{BH}} \sim \frac{\alpha^{\prime}}{G_{5}} r_{w} \cosh \sigma_{p}, \quad M \sim \frac{r_{w}^{2}}{G_{5}} .
$$

Before going into the details of the comparison, we must note a number of peculiarities that arise in the correspondence between near extremal black holes and fundamental strings. The way the extremal limit is reached in the black hole side requires sending $r_{0}^{2} / G_{5} \rightarrow 0$ and $\sigma_{w} \rightarrow \infty$ while keeping $r_{0}^{2} \sinh 2 \sigma_{w} / G_{5}$ (i.e., $n_{w}$ ) fixed. Additionally, one can also send $\sigma_{p} \rightarrow \infty$, keeping $\sigma_{w}-\sigma_{p}$ fixed, thus obtaining an extremal black hole with two charges $\left(n_{w}\right.$ and $\left.n_{p}\right){ }^{6}$ This means that if we want to keep $r_{0} \sim \sqrt{\alpha^{\prime}}$, then the extremal limit corresponds to taking the string coupling $g \rightarrow \infty$. Nevertheless, we can still consider near extremal regimes at weak coupling. The reason is that the mass of the extremal black hole is $M_{\text {ext }} \sim r_{w}^{2} / G_{5}$, whereas the energy above extremality is $\Delta E \sim r_{0}^{2} / G_{5} \cdot{ }^{7}$ Then

$$
\frac{\Delta E}{M_{\mathrm{ext}}} \sim \frac{r_{0}^{2}}{r_{w}^{2}} \ll 1
$$

is small independently of the coupling, and it is in this sense that we talk about a near extremal black hole. Therefore, there is no problem in a black hole close to extremality evolving into a weakly coupled string. We should keep in mind, though, that at weak coupling $\Delta E$ is not infinitesimally small (in string units) and thus the configuration is a finite distance away from the extremal one.

Another important effect in near extremal configurations is that the gravitational dressing can be rather large even after the transition to the weakly coupled string [19]. This comes about by the fact that near the horizon the factors $f_{w}$, $f_{p}$ in the metric are big $\left(\sim \cosh ^{2} \sigma_{w}, \cosh ^{2} \sigma_{p}\right)$, and thereby induce redshifts in quantities like the compact radius $R$ or the free energy above the rest mass with respect to their asymptotic values. Nevertheless, Horowitz and Polchinski have argued that the calculation of the string entropy (3.4) is not affected by the use of the asymptotic values instead of those read from the corrected local metric.

Such redshifts can be read from Eq. (3.2). For example, the local temperature at the string is related to the temperature measured at asymptotic infinity as

$$
T^{(\text {loc })}=\cosh \sigma_{w} \cosh \sigma_{p} T^{(\text {as })} .
$$

The frequency of quanta emitted by the string undergoes a similar redshift when they reach the asymptotically flat region. Therefore, the quotient

$$
\left(\frac{\omega}{T}\right)^{(\text {loc })}=\left(\frac{\omega}{T}\right)^{(\text {as })},
$$

which appears in greybody factors, remains unchanged. ${ }^{8}$

\footnotetext{
${ }^{6}$ The extremal black hole with a single type of charge corresponds to a nondegenerate string state, $N_{R}=N_{L}=0$, with zero entropy. If there are two charges, then $N_{R}=0, N_{L} \neq 0$.

${ }^{7}$ Or, possibly, $\Delta E \sim r_{0}^{2} \cosh 2 \sigma_{p} / G_{5}$. This does not affect the argument.

${ }^{8}$ At this point we admit to have found some difficulty on how to account unambiguously for the detailed effect of the redshift when matching black hole and string parameters. For example, it is not clear to us how the left- and right-moving momenta $p_{R, L}=n_{p} / R \pm n_{w} R / \alpha^{\prime}$ should be redshifted. For the purposes of this paper, we will find a simple way to formulate our arguments that seems to be free of any such ambiguities.
} 
Turn now to analyze the absorption of a neutral scalar of frequency $\omega$ by a fundamental string. The increment in oscillator level due to absorption of neutral quanta is the same for right and left movers, $\delta N_{R}=\delta N_{L}$. Given that the energy increase is $\omega$, the mass shell condition (3.1) yields

$$
\delta N_{R, L} \sim \alpha^{\prime} M \omega
$$

The absorption rate is to be averaged over a statistical ensemble of initial states peaked at a given mass. The emission rate, from which the absorption rate can be obtained, has been computed in [18] using string perturbation theory. The result is

$$
\sigma_{\mathrm{abs}} \sim \frac{G_{5}\left(\delta N_{L}\right)^{2}}{\alpha^{\prime} M \omega} \frac{e^{\beta_{L}^{*} \delta N_{L}+\beta_{R}^{*} \delta N_{R}-1}}{\left(e^{\beta_{L}^{*} \delta N_{L}}-1\right)\left(e^{\beta_{R}^{*} \delta N_{R}}-1\right)},
$$

with

$$
\beta_{R, L}^{*} \equiv \frac{\partial S_{\mathrm{st}}}{\partial N_{R, L}} .
$$

Using Eqs. (3.4), (3.9), and (3.11) the factors in the exponentials are

$$
\begin{aligned}
\beta_{R, L}^{*} \delta N_{R, L} & \sim \frac{\alpha^{\prime} M \omega}{\sqrt{N_{R, L}}} \\
& \sim \frac{\omega}{T_{R, L}^{(\mathrm{st})}},
\end{aligned}
$$

where the last relation can be taken as a definition of the leftand right-moving oscillator effective temperatures. ${ }^{9}$ It is important to notice that the frequency here is measured at the local position of the string. Similarly, $T_{R, L}^{(\text {st) }}$ are local quantities as well.

An assumption needed to derive the result (3.10) is that the Compton wavelength of the scalar be much bigger than the string scale:

$$
\omega \sqrt{\alpha^{\prime}} \ll 1
$$

At the matching point, $r_{0} \sim \sqrt{\alpha^{\prime}}$, this condition is, in fact, less restrictive than the one imposed on the semiclassical calculation.

Consider first the leading term in the very low frequency limit. By expanding Eq. (3.10), and using the expressions for $\delta N_{R, L}(3.9),(3.12)$, one finds

\footnotetext{
${ }^{9}$ Since eventually we are only interested in comparing the functional form of the greybody factors, we do not need to interpret $T_{R, L}^{\text {(st) }}$ as actual temperatures. In fact, we could even do without them. But recall that for a weakly coupled closed string the left- and rightmoving oscillations behave independently of each other, being only related by the mass shell condition. The total degeneracy of the string is the sum of the degeneracies $S_{R, L} \sim \sqrt{N_{R, L}}$ of each separate ensemble, so we could define effective left and right temperatures by $1 / T_{R, L}^{(s t)} \sim\left(\partial S_{R, L} / \partial N_{R, L}\right)\left(\delta N_{R, L} / \delta M\right)$. These coincide with Eq. (3.12).
}

$$
\begin{aligned}
\sigma_{\mathrm{abs}} & \sim G_{5} \frac{\beta_{R}^{*}+\beta_{L}^{*}}{\beta_{R}^{*} \beta_{L}^{*}} \\
& \sim G_{5}\left(\sqrt{N_{R}}+\sqrt{N_{L}}\right) \\
& \sim A_{\mathrm{BH}},
\end{aligned}
$$

thus correctly reproducing the semiclassical leading order result. The proportionality, with a factor of order 1 , between $\sigma_{\text {abs }}$ and the black hole area was found in [18]. The fact that the agreement is not precise is something that the correspondence principle allows for.

As mentioned above, in the quantities tested so far only the particular combination of oscillator level numbers that yields the entropy, or area, enters, so that we have not been able to discern the individual values of the left- and rightmoving oscillator levels. The greybody factors, which depend solely on the quantities $\omega / T_{R, L}$, can convey such information. In this respect, the formal similarity between the perturbative string and classical black hole results, Eqs. (2.23) and (3.10), is most remarkable. Unfortunately, we can easily see that the left- and right-moving temperatures read from the black hole absorption spectrum cannot agree with those obtained from the string spectrum. To this effect, first we must redshift the asymptotic temperatures (2.22) (with $r_{1}=r_{w}, \sigma_{2}=\sigma_{p}$, and $\left.\sigma_{3}=0\right)$ to the location of the string $r \sim r_{0} \sim \sqrt{\alpha^{\prime}}$. This yields

$$
T_{R, L}^{(\mathrm{loc})} \sim \frac{1}{\sqrt{\alpha^{\prime}}},
$$

i.e., both temperatures are of the order of the Hagedorn temperature. Suppose now that they were equal, up to factors of order 1, to the local string temperatures obtained from Eq. (3.12). This would translate into $\sqrt{N_{R}} \sim \sqrt{N_{L}} \sim \sqrt{\alpha^{\prime}} M$, for any values of the charges (requiring only large $\sigma_{w}$ ). But if we take Eq. (3.5) into account, we find that this condition is incompatible with the entropy being given by $S \sim \sqrt{N_{R}}+\sqrt{N_{L}}$.

The conclusion follows that temperatures cannot match and therefore string and black hole greybody factors disagree in their functional dependence. The discrepancy is most patent when $r_{w} \gg r_{p} \gg 1$, but is true throughout virtually all of the parameter range we can probe. ${ }^{10}$ Additionally, when there is essentially one large charge, and $\omega / T$ becomes small, the logarithmic corrections in Eq. (2.24) become another source of trouble.

The same sort of discrepancy appears when comparing the left- and right-moving temperatures for the fourdimensional black hole, though in this case the parameter range is more restricted. Finally, the wave equation for black holes in dimensions higher than five cannot be solved, close to the horizon, in terms of hypergeometric functions, and therefore we do not know how to obtain greybody factors

\footnotetext{
${ }^{10}$ It is perhaps worthwhile noting that the disagreement would also be present if we had not redshifted the asymptotic temperatures to their local values.
} 
such as those in Eq. (2.23). However, in light of the results we have found it would not be surprising if the disagreement persisted in $D \geqslant 6$.

\section{DISCUSSION}

Several comments are in order regarding the discordant result found in the previous section. First of all, one should note that it does not disprove the correspondence principle. At the low frequencies we are working, the greybody corrections to the absorption rates are either of order 1 [when there are two large charges and Eq. (2.23) is valid] or subleading [with just one large charge, Eq. (2.24)]. The modulation in frequency in the former case is not big. Therefore, as far as the absorption rates are concerned, there is no large change throughout the transition from the black hole to the weakly coupled string.

Nevertheless, our result places a limit on the applicability of the simplest model for the correspondence, which thus becomes comparatively less powerful than the "effective string" model for $D$-brane black holes. It appears that the low frequency corrections to the absorption rates undergo seemingly significant changes in the transition from the black hole to the string. One would expect a more detailed examination of the correspondence to reveal the reason. Apparently, the lowest order perturbative string result (amplitudes on the sphere) cannot account for this. One-loop corrections to the closed string vertex should add terms $\propto g^{2}$. On the other hand, in the regime where only one of the charges is large, the correction term resulting from expanding the greybody factors is

$$
\frac{\omega^{2}}{T_{R} T_{L}} \propto g^{2} \omega^{2},
$$

but recall that this is shadowed by the logarithmic correction in Eq. (2.24) (which is $\propto g^{2}$, too). Things are further complicated by the fact that in four dimensions the leading term at small frequencies goes like $g^{2} \omega$. It is very unclear whether string vertex corrections can simultaneously account for all these facts.

Apart from the perturbative corrections (higher powers of $g$ ) to the emission spectrum of the string, another source for possible corrections of the correspondence comes from string-size $\left(\alpha^{\prime}\right)$ effects on the black hole geometry. However, these do not seem to be important to account for the area (entropy) of the black hole horizon (this is part of the content of the correspondence principle), so it is uncertain whether they might significantly alter the role of the horizon as a boundary for scattering wave functions. Also, such corrections are presumably very hard to compute.

Actually, there are reasons to suspect that the NS black hole-string correspondence might not be so simple for black holes close to extremality. From what we have seen, if we keep the horizon at a string scale size, the BPS limit is only reached for $g \rightarrow \infty$. Thus, a near-extremal black hole that evolves into a weakly coupled string might not be close to the BPS state $N_{R}=0$. This is in contrast with the situation for the black hole with three nonvanishing charges [4,5,7], where a state can remain infinitesimally close to extremality throughout the passage from strong to weak coupling. For the case we study here, with only two charges, the weakly coupled state is instead always a finite distance away from the extremal state.

This is very presumably related to the lack of agreement of radiation profiles. Consider a string that is close to the BPS state; we would expect this condition to mean $N_{L} \gg N_{R}$. In turn, this would mean $T_{L}^{(\mathrm{st})} \gg T_{R}^{(\mathrm{st})}$, which can hardly be harmonized with the classical black hole result, which requires $T_{L}=T_{R}$ for all values of the parameters (near extremality). Indeed, it is not likely that $T_{L} \gg T_{R}$ can hold at the transition point. The reason is that the Hawking temperature is related to the left and right temperatures by

$$
\frac{2}{T_{H}}=\frac{1}{T_{L}}+\frac{1}{T_{R}} .
$$

A similar relation also holds for the temperatures defined from the string spectrum. It implies that, if one of the temperatures is much higher than the other, say, $T_{L} \gg T_{R}$, then $T_{R} \sim T_{H} \ll T_{L}$. But, as we have seen, the gravitational dressing turns the asymptotic temperature of the radiation $T_{H}$ into a local string temperature $1 / \sqrt{\alpha^{\prime}}$ at the matching point, and therefore we would have $T_{L}^{(\text {loc })}$ much larger than the string scale, which does not seem reasonable. A more conceivable scenario would be that, as the black hole shrinks to the string scale, the number of left moving oscillators $N_{L}$ can never reach a value much bigger than $N_{R}$, in the sense that we do not get $N_{L} / N_{R} \gg 1$, though the difference $N_{L}-N_{R}=n_{p} n_{w}$, which is kept fixed, can still be quite large. Such a string would not be close to the BPS state. It would have $T_{R}^{(\mathrm{st})} \sim T_{L}^{\text {(st) }} \sim 1 / \sqrt{\alpha^{\prime}}$, and could possibly agree with the left and right black hole temperatures.

There does not appear to be any simple enough way to implement this picture. A complete analysis should involve a proper treatment of the locally corrected quantities. However, this is unlikely to be enough for solving the puzzle, at least within the simplest model for black hole-string correspondence (i.e., that without higher order corrections to the string vertex or the black hole geometry). The reason is that our result in the previous section seems to be largely independent of how the gravitational dressing acts. The redshift effect does not seem suffice to obtain $T_{L}^{(\mathrm{st})} \sim T_{R}^{\text {(st) }}$, and simultaneously preserve the agreement between string and black hole entropies. The conclusion seems to be that, at least in this region of black hole parameter space, the detailed correspondence to fundamental strings is not simple.

As an aside, in view of the results above, we find it remarkable, though somewhat puzzling, that if these same fundamental strings are placed in the background of a magnetic NS fivebrane, then precise agreement can be found both for the entropies and the emission rates [33]. Apparently, the only effect of the fivebrane on the string is to rescale the oscillator number and string tension, and restrict the motion of the string to the world volume of the fivebrane. Otherwise, the dynamics of the string is unaltered. The resulting black hole, on the other hand, possesses now a regular horizon in 
the extremal limit. The correspondence principle, however, cannot be applied to these configurations, since their horizon does not decrease below the string size at weak coupling.

It is perhaps disappointing to find that the simplest approximation to the black hole-string correspondence does not seem to work as well as it does for other kinds of black holes, namely, those with a regular extremal limit. There are number of other sorts of scalars (charged, fixed, intermediate [8]) that can be used to probe further the correspondence between black holes and fundamental strings, and may shed further light on the problem. This is currently under investigation.

\section{ACKNOWLEDGMENTS}

We are indebted to Gary Horowitz and John Pierre for useful conversations on these issues. We also thank Arvind Rajaraman for pointing out an error in a previous version of this paper and for making his notes available [26]. This work was partially supported by the FPI (MEC-Spain) and by Grant No UPV 063.310-EB225/95.
[1] A. Strominger and C. Vafa, Phys. Lett. B 379, 99 (1996).

[2] G. T. Horowitz, "The Origin of Black Hole Entropy in String Theory,' Proceedings of the Pacific Conference on Gravity and Cosmology, Seoul, Korea, 1996 (unpublished) gr-qc/9604051.

[3] J. Maldacena, "Black Holes in String Theory," hep-th/9607235.

[4] C. G. Callan and J. Maldacena, Nucl. Phys. B472, 591 (1996).

[5] G. T. Horowitz and A. Strominger, Phys. Rev. Lett. 77, 2368 (1996).

[6] S. R. Das and S. D. Mathur, Nucl. Phys. B478, 561 (1996).

[7] J. Maldacena and A. Strominger, Phys. Rev. D 55, 861 (1997).

[8] S. S. Gubser and I. R. Klebanov, Nucl. Phys. B482, 173 (1996); Phys. Rev. Lett. 77, 4491 (1996); C. G. Callan, S. S. Gubser, I. R. Klebanov, and A. A. Tseytlin, Nucl. Phys. B489, 65 (1997); I. R. Klebanov and M. Krasnitz, Phys. Rev. D 55, 3250 (1997); I. R. Klebanov, A. Rajaraman, and A. A. Tseytlin, hep-th/9704112.

[9] F. Dowker, D. Kastor, and J. Traschen, hep-th/9702109.

[10] S. W. Hawking and M. M. Taylor-Robinson, Phys. Rev. D 55, 7680 (1997).

[11] S. de Alwis and K. Sato, Phys. Rev. D 55, 6181 (1997).

[12] J. M. Maldacena, Phys. Rev. D 55, 7645 (1997).

[13] J. M. Maldacena, Nucl. Phys. B477, 168 (1996).

[14] I. R. Klebanov and S. D. Mathur, hep-th/9701187.

[15] G. T. Horowitz, J. Maldacena, and A. Strominger, Phys. Lett. B 383, 151 (1996).
[16] L. Susskind, RU-9344, hep-th/9309145.

[17] E. Halyo, A. Rajaraman, and L. Susskind, Phys. Lett. B 392, 319 (1997).

[18] E. Halyo, B. Kol, A. Rajaraman, and L. Susskind, Phys. Lett. B 401, 15 (1997).

[19] G. T. Horowitz and J. Polchinski, Phys. Rev. D 55, 6189 (1997).

[20] F. Larsen, Phys. Rev. D 56, 1005 (1997).

[21] M. Cvetic and D. Youm, Nucl. Phys. B476, 118 (1996).

[22] A. A. Starobinski and S. M. Churilov, Sov. Phys. JETP 38, 1 (1974).

[23] S. A. Teukolsky and W. H. Press, Astrophys. J. 193, 443 (1974).

[24] D. N. Page, Phys. Rev. D 13, 198 (1976).

[25] W. G. Unruh, Phys. Rev. D 14, 325 (1976).

[26] A. Rajaraman (unpublished).

[27] G. W. Gibbons, Commun. Math. Phys. 44, 245 (1975).

[28] S. R. Das, G. W. Gibbons, and S. D. Mathur, Phys. Rev. Lett. 78, 417 (1997).

[29] S. Das, A. Dasgupta, and T. Sarkar, Phys. Rev. D 55, 7693 (1997).

[30] A. Sen, Nucl. Phys. B440, 421 (1995).

[31] A. Sen, Mod. Phys. Lett. A 10, 2081 (1995).

[32] G. T. Horowitz and A. Strominger, Nucl. Phys. B360, 197 (1991); J. Horne, G. T. Horowitz, and A. Steif, Phys. Rev. Lett. 68, 568 (1992).

[33] E. Halyo, hep-th/9610068. 